\title{
Patients knowledge of and attitudes to antibiotics use in Saudi hospitals: The institutional role of patients education
}

Saleh Alghamdi a, Eshtyag Bajnaid b, Zoe Aslanpour a, Nada Shebl a, Ilhem Berrou c

a Department of Pharmacy, Pharmacology and Post-Graduate Medicine, Division of Postgraduate Pharmacy Practice and Public Health, University of Hertfordshire, College Lane Campus, Hatfield, Herts, AL10 9AB, The United Kingdom

b Head of Clinical Pharmacy Department, Pharmaceutical Services Administration, King Abdullah Medical City, Makkah, Saudi Arabia

${ }^{\mathrm{C}}$ Department of Nursing \& Midwifery, University of the West of England, Bristol, BS16 1DD, The United Kingdom

\section{Introduction \& Aims}

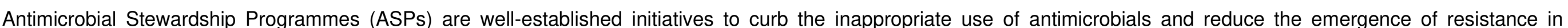

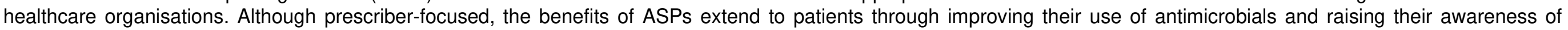

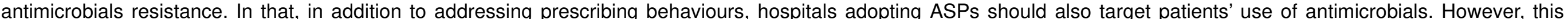

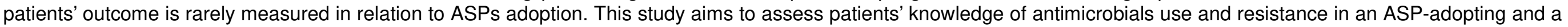
non-adopting hospital to evaluate the institutional role of patients' education as part of ASP.

\section{Methodology}

This is a cross-sectional study by means of a self-administered patient's questionnaire in two Saudi hospitals; one with ASP, and one which is yet to adopt ASP. Patients' responses were collected from May to August 2017. Data analysis included descriptive and correlation statistics.

\section{Results \& Discussion}

176 patients responded to the survey. $62 \%$ of the responses were from the non-ASP adopting hospital. Patients were mainly $31-40$ years of age (46\%). We identified a comparable lack of knowledge of appropriate antimicrobials use in both hospitals such that $68 \%$ of participants agreed to share their antibiotics with family members, and only $31 \%$ of participants believe that antibiotic resistance can result from not completing the full course of antibiotics. Further, only $26 \%$ of patients think that antibiotic resistance is a problem in Saudi today, and only $27 \%$ believe that their hospital stay improved their awareness of antibiotic resistance.

\section{Conclusion(s)}

Patients awareness of appropriate antimicrobial use and resistance is low in Saudi hospitals. Successful adoption of ASPs should ensure that patients are counselled on correct antimicrobial use, and are aware of their potential contribution to antimicrobial resistance. Pharmacists can be more involved in patients counselling to ensure ASPs adoption outcomes are achieved.

\section{Acknowledgements}

I would like to express my special appreciation and thanks to my supervisors; Dr Zoe Aslanpour, Dr Ilhem Berrou, \& Dr Nada Atef Shebl. I would like also to thank AlBaha University in Saudi Arabia for sponsoring my PhD project.

\section{References}

Centers for Disease Control and Prevention. (2014). Core Elements of Hospital Antibiotic Stewardship Programs. US Department of Health and Human Services, $C D C, 1-25$. Retrieved from http://www.cdc.gov/getsmart/healthcare/rimplementation/core-elements.htm/

World Health Organization. (2015). WHO report finds systems to combat antibiotic resistance lacking. Retrieved May 7, 2015, from http://www.who.int/mediacentre/news/releases/2015/antibiotic-resistance-lacking/en/ 